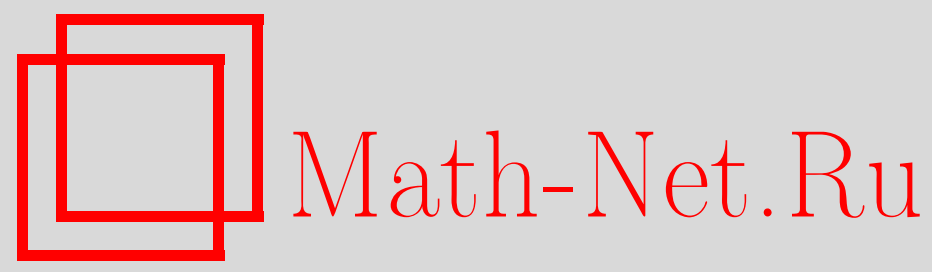

У. Гогинава, О чезаровских средних двойных тригонометрических рядов Фурье, Матем. заметки, 2003, том 74, выпуск 4, 502-507

DOI: https://doi.org/10.4213/mzm285

Использование Общероссийского математического портала Math-Net.Ru подразумевает, что вы прочитали и согласны с пользовательским соглашением http://www . mathnet.ru/rus/agreement

Параметры загрузки:

IP : 35.174 .16 .151

26 апреля 2023 г., $11: 11: 14$

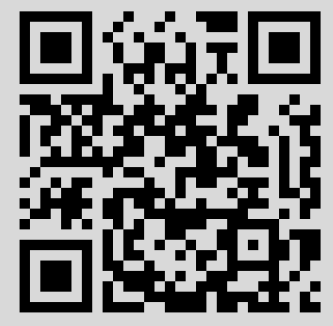




\section{О ЧЕЗАРОВСКИХ СРЕДНИХ ДВОЙНЫХ ТРИГОНОМЕТРИЧЕСКИХ РЯДОВ ФУРЬЕ}

\section{У. Гогинава}

Жижиашвили изучил вопросы, связанные с аппроксимативными свойствами Чезаровских средних тригонометрических рядов Фурье для функций двух переменных в пространствах $H^{\omega}$. Нами доказывается, что эту оценку нельзя улучшить при $p=1$ или $p=\infty$.

Библиография: 5 названий.

Скажем, что функция $f(x, y)$ принадлежит $L^{p}, 1 \leqslant p<+\infty$, если $f$ является $2 \pi$-периодической по каждой переменной, измеримой на $[0,2 \pi)^{2}$ функцией, для которой конечен функционал

$$
\|f\|_{p}=\left\{\int_{0}^{2 \pi} \int_{0}^{2 \pi}|f(x, y)|^{p} d x d y\right\}^{1 / p},
$$

причем, если $p=+\infty$, то пространство $L^{\infty}$ будем считать совпадающим с пространством $2 \pi$-периодических непрерывных функций двух переменных $C$.

Для функции $f(x, y)$, принадлежащей $L^{p}$, выражения

$$
\omega_{1}(\delta, f)_{p}=\sup _{|h| \leqslant \delta}\|f(\cdot+h, \cdot)-f(\cdot, \cdot)\|_{p}
$$

и

$$
\omega_{2}(\delta, f)_{p}=\sup _{|h| \leqslant \delta}\|f(\cdot, \cdot+h)-f(\cdot, \cdot)\|_{p}
$$

назьвают частными $L^{p}$-модулями непрерывности функции $f$.

Пусть $\omega$ - модуль непрерывности (см. [1]). Если $p \in[1,+\infty]$, то будем предполагать, что

$$
H_{p}^{\omega}=\left\{f: \omega_{i}(\delta, f) \leqslant A(f, p) \omega(\delta), \delta \in[0, \pi], i=1,2\right\}
$$

где $A(f, p)$ - положительная константа.

Пусть $f \in L^{1}$. Ее рядом Фурье по тригонометрической системе называется ряд

$$
\sum_{m, n=-\infty}^{+\infty} C_{m, n} e^{i m x} e^{i n y}
$$

где

$$
C_{m, n}=\frac{1}{4 \pi^{2}} \int_{0}^{2 \pi} \int_{0}^{2 \pi} f(u, v) e^{-i m u} e^{-i n v} d u d v
$$

суть коэффициенты Фурье функции $f$. 
Пусть $\alpha \in(-1,0) \cup(0,+\infty)$ - некоторое число. Выражения

$$
\sigma_{m}^{\alpha}(f ; x)=\frac{1}{A_{m}^{\alpha}} \sum_{j=0}^{m} A_{m-j}^{\alpha-1} S_{j, j}(f, x)
$$

назьвают $(C, \alpha)$-средними рядов $(1)$, где $S_{j, j}(f, x)-j$-я квадратная частная сумма ряда $(1)$, a

$$
A_{0}^{\alpha}=1, \quad A_{k}^{\alpha}=\frac{(\alpha+1) \cdots(\alpha+k)}{k !}, \quad k=1,2, \ldots
$$

Известно (см. Зигмунд [2, с. 131]), что

$$
c_{1}(\alpha) k^{\alpha} \leqslant A_{k}^{\alpha} \leqslant c_{2}(\alpha) k^{\alpha}
$$

Жижиашвили ([3, с. 367-381]) изучил вопросы, связанные с апшроксимативньми свойствами $(C, \alpha)$-средних, $\alpha \in(-1,0) \cup(0,+\infty)$, рядов $(1)$ в пространствах $L^{p}$, $p \in[1,+\infty]$. В частности, он доказал, что справедлива следующая

Tеорема А. Пусть $\alpha \in(-1,0) \cup(0,+\infty)$. Предположим, что функиия $f \in L^{p}$ при некотором $p \in[1,+\infty]$. Тогда

a) если $\alpha \in(0,+\infty)$, то

$$
\left\|\sigma_{m}^{\alpha}(f)-f\right\|_{p} \leqslant A(\alpha, p) \frac{1}{m}\left[\int_{1 / m}^{\pi} \frac{\omega_{1}\left(s_{1}, f\right)_{p}}{s_{1}^{2}} d s_{1}+\int_{1 / m}^{\pi} \frac{\omega_{2}\left(s_{2}, f\right)_{p}}{s_{2}^{2}} d s_{2}\right]
$$

б) если же $\alpha \in(0,1)$, mo

$$
\left\|\sigma_{m}^{-\alpha}(f)-f\right\|_{p} \leqslant A(\alpha, p) m^{\alpha} \log (m+2)\left[\omega_{1}\left(\frac{1}{m}, f\right)_{p}+\omega_{2}\left(\frac{1}{m}, f\right)_{p}\right]
$$

Там же доказано, что неравенство (3) при $p=1$ или $p=\infty$ точно для всего пространства $L^{p}$.

Мы утверждаем, что при выполнении условия

$$
\frac{\omega(\delta)}{\delta} \uparrow \infty, \quad \delta \rightarrow 0+
$$

неравенство (4) нельзя улучшить на всем классе $H_{p}^{\omega}$ при $p=1$ или $p=\infty$. В частности, справедлива следующая

ТЕОРема 1. Пусть $\alpha \in(0,1)$ и модуль непрерывности $\omega(\delta)$ удовлетворяет условию (5). Тогда при $p=\infty$ или $p=1$ в классе $H_{p}^{\omega}$ существует функция $f$, зависящая от $p$, такая, что

$$
\varlimsup_{n \rightarrow \infty} \frac{\left\|\sigma_{n}^{-\alpha}(f)-f\right\|_{p}}{\omega(1 / n) n^{\alpha} \log n}>0 .
$$

Из неравенства (4) вытекает (см. [3, с. 379]) аналог теоремы Зигмунда (см. [4]) для средних $\sigma_{n}^{-\alpha}$. В частности, имеет место следующая 
Теорема Б. Пусть $f \in H_{p}^{\delta^{\alpha}}$ при некотором $\alpha \in(0,1)$. Тогда для любого $\beta \in(0, \alpha)$ справедливо следующее соотношение:

$$
\left\|\sigma_{m}^{-\beta}(f)-f\right\|_{p} \rightarrow 0, \quad m \rightarrow \infty .
$$

Из теоремы 1 следует, что при $\beta=\alpha$ теорема Б теряет силу для $p=+\infty$ и $p=1$, а именно, имеет место следующая

Теорема 2. Пусть $\alpha \in(0,1)$ - некоторое число. Тогда при $p=\infty$ или $p=1$ в классе $H_{p}^{\delta^{\alpha}}$ сущ,ествует функиия $f$, зависящая от $p$, такая, что

$$
\varlimsup_{n \rightarrow \infty}\left\|\sigma_{n}^{-\alpha}(f)-f\right\|_{p}=\infty
$$

ДокАЗАТЕЛЬСТво тЕОРЕМЫ 1. Вначале докажем теорему 1 для случая $p=\infty$. Пусть последовательность натуральных чисел $\left\{n_{k}: k \geqslant 1\right\}$ такова, что

$$
\begin{aligned}
\omega\left(\frac{1}{n_{k+1}}\right) & \leqslant \frac{1}{2} \omega\left(\frac{1}{n_{k}}\right), \\
\sum_{j=1}^{k} \omega\left(\frac{1}{n_{j}}\right) n_{j} & \leqslant \omega\left(\frac{1}{n_{k+1}}\right) n_{k+1}, \\
k n_{k} \omega\left(\frac{1}{n_{k}}\right) & \leqslant n_{k+1} \omega\left(\frac{1}{n_{k+1}}\right), \\
3 n_{k} & <n_{k+1} .
\end{aligned}
$$

Определим функцию $f_{0}(x, y)$ формулой

$$
f_{0}(x, y)=\sum_{k=1}^{\infty} f_{k}(x, y)
$$

где

$$
f_{k}(x, y)=\omega\left(\frac{1}{n_{k}}\right) \cos n_{k} x \sin n_{k} y, \quad \text { если } x \in[-\pi, \pi], \quad y \in[0, \pi]
$$

и

$$
f_{k}(x,-y)=f_{k}(x, y), \quad f_{k}(x+2 \pi, y)=f_{k}(x, y+2 \pi)=f_{k}(x, y) .
$$

Из (6) легко видеть, что $f_{0} \in C$. Докажем, что $f_{0} \in H_{\infty}^{\omega}$. Для любого $\delta>0$ выберем числа $n_{s}$ и $n_{s+1}$ из последовательности $\left\{n_{k}: k \geqslant 1\right\}$ так, что $1 / n_{s+1} \leqslant \delta<1 / n_{s}$. Тогда, так как $\omega\left(\delta_{1}\right) / \delta_{1} \leqslant 2 \omega\left(\delta_{2}\right) / \delta_{2}, 0<\delta_{2}<\delta_{1}$, то из (6) и (7) получим

$$
\begin{aligned}
\left|f_{0}(x+\delta, y)-f_{0}(x, y)\right| & =O\left(\sum_{k=1}^{s} \omega\left(\frac{1}{n_{k}}\right) n_{k} \delta+\sum_{k=s+1}^{\infty} \omega\left(\frac{1}{n_{k}}\right)\right) \\
& =O\left(\omega\left(\frac{1}{n_{s}}\right) n_{s} \delta+\omega\left(\frac{1}{n_{s+1}}\right)\right)=O(\omega(\delta)) .
\end{aligned}
$$

Аналогично доказьвается, что $\omega_{2}(\delta, f)=O(\omega(\delta))$, т.е. $f_{0} \in H_{\infty}^{\omega}$. 
Легко видеть, что

$$
\begin{aligned}
\left|\sigma_{n_{k}}^{-\alpha}\left(f_{0} ; 0,0\right)-f_{0}(0,0)\right| \geqslant \mid & \sigma_{n_{k}}^{-\alpha}\left(f_{k} ; 0,0\right)|-| \sigma_{n_{k}}^{-\alpha}\left(\sum_{j=k+1}^{\infty} f_{j} ; 0,0\right) \mid \\
& -\left|\sigma_{n_{k}}^{-\alpha}\left(\sum_{j=1}^{k-1} f_{j} ; 0,0\right)\right|=I-I I-I I I .
\end{aligned}
$$

Tak как

$$
\begin{aligned}
\left|\int_{0}^{\pi} D_{n_{k}}(v) \sin n_{k} v d v\right| & \geqslant\left|\int_{\pi / n_{k}}^{\pi} \frac{\sin ^{2} n_{k} v}{2 \sin (v / 2)} \cos \frac{v}{2} d v\right|-O(1) \\
& \geqslant c \int_{\pi / n_{k}}^{\pi / 2} \frac{1}{v} d v-O(1) \geqslant c \log n_{k}
\end{aligned}
$$

то из (2) имеем

$$
\begin{aligned}
I & =\frac{4}{\pi^{2}} \frac{\omega\left(1 / n_{k}\right)}{A_{n_{k}}^{-\alpha}}\left|\sum_{j=0}^{n_{k}} A_{n_{k}-j}^{-\alpha-1} \int_{0}^{\pi} D_{j}(v) \sin n_{k} v d v \int_{0}^{\pi} D_{j}(u) \cos n_{k} u d u\right| \\
& =\frac{2}{\pi} \frac{\omega\left(1 / n_{k}\right)}{A_{n_{k}}^{-\alpha}}\left|\int_{0}^{\pi} D_{n_{k}}(v) \sin n_{k} v d v\right| \geqslant c \omega\left(\frac{1}{n_{k}}\right) n_{k}^{\alpha} \log n_{k} .
\end{aligned}
$$

Легко видеть, что

$$
I I=0 .
$$

Tak как

$$
\omega_{s}\left(f_{j}, \frac{1}{n_{k}}\right)=O\left(\omega\left(\frac{1}{n_{j}}\right) \frac{n_{j}}{n_{k}}\right) \quad \text { при } s=1,2,
$$

то из (4) и (8) имеем

$$
\begin{aligned}
I I I & \leqslant \sum_{j=1}^{k-1}\left\|\sigma_{n_{k}}^{-\alpha}\left(f_{j}\right)-f_{j}\right\|_{C}=O\left(n_{k}^{\alpha} \log n_{k} \sum_{j=1}^{k-1}\left(\omega_{1}\left(f_{j}, \frac{1}{n_{k}}\right)+\omega_{2}\left(f_{j}, \frac{1}{n_{k}}\right)\right)\right) \\
& =O\left(n_{k}^{\alpha-1} \log n_{k} \sum_{j=1}^{k-1} \omega\left(\frac{1}{n_{j}}\right) n_{j}\right)=O\left(n_{k}^{\alpha-1} \log n_{k} \omega\left(\frac{1}{n_{k-1}}\right) n_{k-1}\right) .
\end{aligned}
$$

Из (10)-(13) следует справедливость теоремы 1 при $p=\infty$. Теперь докажем теорему при $p=1$. Для этого воспользуемся следуюшими утверждениями.

ТЕОРемА В (Зигмунд [5]). Если $T_{n}(x)$ - тригонометрический полином порядка $n$, то для любого $p \geqslant 1$

$$
\left\|T_{n}^{\prime}\right\|_{p} \leqslant n\left\|T_{n}\right\|_{p}
$$


ЛЕмма А (Жижиашвили [3]). Если $n$ - произвольное натуральное число, а $k$ произвольное неотрицательное челое число, то

$$
\frac{1}{2 \pi} \int_{0}^{2 \pi}|V(n, k, x)| d x \leqslant 1, \quad \int_{0}^{2 \pi}\left|V_{2}(n, x)\right| d x \geqslant c \ln n,
$$

әде

$$
\begin{aligned}
V(n, k, x)= & \frac{1}{n+1}(\cos k x+2 \cos (k+1) x+\cdots+(n+1) \cos (k+n) x \\
& +n \cos (k+n+1) x+\cdots+\cos (k+2 n) x)
\end{aligned}
$$

$u$

$$
V_{2}(n, x)=\frac{1}{n} \sum_{k=1}^{n-1} k \cos (k+n) x .
$$

Определим функцию $g_{0}(x, y)$ формулой

$$
g_{0}(x, y)=\sum_{k=1}^{\infty} \omega\left(\frac{1}{n_{k}}\right) V\left(n_{k}, n_{k}, x\right) V\left(n_{k}, n_{k}, y\right) .
$$

Докажем, что $g \in H_{1}^{\omega}$. Для любого $\delta>0$ подберем числа $n_{s}$ и $n_{s+1}$ из последовательности $\left\{n_{k}: k \geqslant 1\right\}$ так, что $1 / n_{s+1} \leqslant \delta<1 / n_{s}$. Тогда, применяя теорему Лагранжа, теорему В, лемму A, (6) и (7), имеем

$$
\begin{aligned}
\int_{0}^{2 \pi} & \int_{0}^{2 \pi}\left|g_{0}(x+\delta, y)-g_{0}(x, y)\right| d x d y \\
\leqslant & \sum_{k=1}^{s} \omega\left(\frac{1}{n_{k}}\right) \int_{0}^{2 \pi} \int_{0}^{2 \pi}\left|V\left(n_{k}, n_{k}, x+\delta\right)-V\left(n_{k}, n_{k}, x\right)\right|\left|V\left(n_{k}, n_{k}, y\right)\right| d x d y \\
& \quad+\sum_{k=s+1}^{\infty} \omega\left(\frac{1}{n_{k}}\right) \int_{0}^{2 \pi} \int_{0}^{2 \pi}\left|V\left(n_{k}, n_{k}, x+\delta\right)-V\left(n_{k}, n_{k}, x\right)\right|\left|V\left(n_{k}, n_{k}, y\right)\right| d x d y \\
\leqslant & 2 \pi \delta \sum_{k=1}^{s} \omega\left(\frac{1}{n_{k}}\right) \int_{0}^{2 \pi}\left|V^{\prime}\left(n_{k}, n_{k}, x+\theta \delta\right)\right| d x+8 \pi^{2} \sum_{k=s+1}^{\infty} \omega\left(\frac{1}{n_{k}}\right) \\
\leqslant & 3(2 \pi)^{2} \delta \sum_{k=1}^{s} \omega\left(\frac{1}{n_{k}}\right) n_{k}+16 \pi^{2} \omega\left(\frac{1}{n_{s+1}}\right) \\
\leqslant & 6(2 \pi)^{2} \omega\left(\frac{1}{n_{s}}\right) n_{s} \delta+16 \pi^{2} \omega(\delta)=O(\omega(\delta)),
\end{aligned}
$$

где $\theta \in(0,1)$. Аналогично доказывается, что $\omega_{2}(\delta, f)=O(\omega(\delta))$, т.е. $f \in H_{1}^{\omega}$.

Учитывая ортогональность тригонометрической системы, из леммы $\mathrm{A},(2)$ и $(9)$ имеем

$$
\begin{aligned}
& \left\|\sigma_{2 n_{k}}^{-\alpha}\left(g_{0}\right)-g_{0}\right\|_{1} \geqslant \int_{0}^{2 \pi}\left|\int_{0}^{2 \pi}\left[\sigma_{2 n_{k}}^{-\alpha}\left(g_{0} ; x, y\right)-g_{0}(x, y)\right] \cos 2 n_{k} x d x\right| d y \\
& \quad \geqslant \int_{0}^{2 \pi}\left|\int_{0}^{2 \pi} \sigma_{2 n_{k}}^{-\alpha}\left(g_{0} ; x, y\right) \cos 2 n_{k} x d x\right| d y-\int_{0}^{2 \pi}\left|\int_{0}^{2 \pi} g_{0}(x, y) \cos 2 n_{k} x d x\right| d y
\end{aligned}
$$




$$
\begin{aligned}
= & \frac{1}{A_{2 n_{k}}^{-\alpha}} \int_{0}^{2 \pi}\left|\int_{0}^{2 \pi} S_{2 n_{k}, 2 n_{k}}\left(g_{0} ; x, y\right) \cos 2 n_{k} x d x\right| d y \\
& -\int_{0}^{2 \pi}\left|\int_{0}^{2 \pi} g_{0}(x, y) \cos 2 n_{k} x d x\right| d y \\
= & \pi \frac{\omega\left(1 / n_{k}\right)}{A_{2 n_{k}}^{-\alpha}} \int_{0}^{2 \pi} \frac{1}{n_{k}+1}\left|\sum_{j=n_{k}}^{2 n_{k}}\left(j-n_{k}+1\right) \cos j y\right| d y \\
& -\pi \omega\left(\frac{1}{n_{k}}\right) \int_{0}^{2 \pi}\left|V\left(n_{k}, n_{k}, y\right)\right| d y \\
\geqslant & \pi \frac{\omega\left(1 / n_{k}\right)}{A_{2 n_{k}}^{-\alpha}} \int_{0}^{2 \pi} \frac{1}{n_{k}+1}\left|\sum_{j=1}^{n_{k}+1} j \cos \left(j+n_{k}-1\right) y\right| d y-2 \pi^{2} \omega\left(\frac{1}{n_{k}}\right) \\
\geqslant & \pi \frac{\omega\left(1 / n_{k}\right)}{A_{2 n_{k}}^{-\alpha}} \frac{n_{k}-1}{n_{k}+1} \int_{0}^{2 \pi}\left|V_{2}\left(n_{k}-1, y\right)\right| d y \\
& -\pi \frac{\omega\left(1 / n_{k}\right)}{A_{2 n_{k}}^{-\alpha}} \frac{1}{n_{k}+1} \int_{0}^{2 \pi} \mid\left(n_{k}-1\right) \cos \left(2 n_{k}-2\right)+n_{k} \cos \left(2 n_{k}-1\right) y \\
& +\left(n_{k}+1\right) \cos 2 n_{k} y \mid d y-2 \pi^{2} \omega\left(\frac{1}{n_{k}}\right) \\
\geqslant & c_{3}(\alpha) \omega\left(\frac{1}{n_{k}}\right) n_{k}^{\alpha} \log n_{k}-c_{4}(\alpha) \omega\left(\frac{1}{n_{k}}\right) n_{k}^{\alpha}-2 \pi^{2} \omega\left(\frac{1}{n_{k}}\right) .
\end{aligned}
$$

Теорема 1 доказана.

\section{СПИСОК ЦИТИРОВАННОЙ ЛИТЕРАТУРЫ}

[1] Никольский С. М. Приближение функций многих переменных и теоремы вложения. М.: Наука, 1977.

[2] Зигмунд А. Тригонометрические ряды. Т. 1. М.: Мир, 1965.

[3] ЖКижиашвили Л.В. Некоторые вопросы теории тригонометрических рядов Фурье и их сопряженных. Тбилиси: Изд. ТГУ, 1993.

[4] Zygmund A. Sur la summabilité des series de Fourier des fonctions verifiant la condition de Lipschitz // Bull. Acad. Polon. Sci. Ser. Math. Astronom. Phys. 1925. P. 1-9.

[5] Zygmund A. A remark on conjugate series // PLMS. 1932. V. 34. P. 392-400.

Тбилисский государственный университет

Поступило им. И. Джавахишвили 25.02 .2002

E-mail : z_goginava@hotmail.com 